\title{
Psicología y Didáctica de las Ciencias de la naturaleza ¿concepciones alternativas?

\author{
JuAN IGNACIO POZO
} \\ Universidad Autónoma de Madrid
}

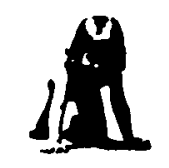

\begin{abstract}
Resumen
El presente trabajo analiza las aportaciones recientes de la Psicología y Didáctica de las Ciencias de la Naturaleza a la investigación del aprendizaje y la enseñanza de las Ciencias. Los puntos de partida y los intereses iniciales de ambas aportaciones son, sin duda, diferentes. Mientras la Psicología ba estado más centrada en los procesos generales del pensamiento científico, la Didáctica ba estudiado sobre todo las «concepciones alternativas» mantenidas por los alumnos en áreas específicas. Pero esta diferente perspectiva no ba impedido la existencia de datos y preocupaciones crecientemente comunes. Estos objetivos comunes estarian relacionados no sólo con los rasgos característicos de lo "ciencia intuitiva» de los alumnos, enraizados en la naturaleza del conocimiento cotidiano estudiado por la psicología cognitiva, sino también con la naturaleza y los mecanismos del cambio conceptual, que debe vincularse a los contextos de uso del conocimiento científico, tanto escolares como cotidianos.

Palabras clave: Constructivismo; Aprendizaje y enseñanza de la ciencia; concepciones alternativas; expertos/novatos; conocimiento cotidiano; cambio conceptual; influencia del contexto.
\end{abstract}

\section{Psychology and Natural Science Didactics: Alternative perspectives?}

\section{Abstract}

This paper analyses recent contributions from Psychology and Natural Science Didactics to research on Science learning and teaching processes. The starting point and initial interest of Psychology and Didactics are undoubtedly different. While Psychology has concentrated on general processes of scientific thought, Didactics has studied students' 'altemative frameworks' on specific areas of the science curriculum. But despite these differences, botb disciplines increasingly share common concerns and research data. These common aims would be related to: the charateristic features of students' 'intuitive science, rooted in the nature of everyday knowledge which Cognitive Psychology studies; the nature and mechanisms of conceptual change, which must be linked to scientific knowledge both in school and everyday contexts.

Key words: Constructivism; Science learning; Science teaching; Alternative frameworks; Expert/novice; Everyday cognition; Conceptual change; Context influence.

Agradecimientos: El presente trabajo es producto de las reflexiones y discusiones habidas durante la realización de dos proyectos de investigación consecutivos financiados por el C.I.D.E. en torno al aprendizaje y comprensión de nociones científicas durante la adolescencia. EI Proyecto recién concluido, «La comprensión de la Química en la adolescencia», ha implicado de hecho un diálogo entre preocupaciones psicológicas y didácticas muy próximo a los objetivos de este artículo. Debo agradecer aquí la colaboración de Miguel Angel Gómez Crespo, Angeles Sanz y María del Puy Pérez, no sólo en dicho Proyecto, sino sobre todo en ayudar a fermentar las suficientes dudas como para que el trabajo de investigación siga teniendo sentido.

Comespondencia con autor: Facultad de Psicología. Universidad Autónoma de Madrid. 28049 Madrid. 


\section{INTRODUCCION: SOBRE LAS MULTIPLES CARAS Y NIVELES DE UNA CONFUSA RELACION}

Las relaciones entre la Psicología y la Didáctica son como aquellos viejos diagramas de Venn que estudiábamos en el colegio. Como se recordará, solían tener una extraña forma de óvalo que evocaba vagamente a un pulmón y, ante todo, se les reconocía por aquella curiosa costumbre suya de unirse y separarse, solaparse y superponerse, siguiendo un confuso ritual que no siempre acabábamos por entender. Otro tanto parece sucederles a la Psicología y la Didáctica de las Ciencias. Según cómo y desde dónde las miremos, aparecen como óvalos superpuestos, conjuntos disjuntos o extraños pulmones respirando desacompasadamente. La mirada que aquí ofreceremos sobre esta tierra de nadie -y por tanto de todos- que es el punto de encuentro entre la Psicología y la Didáctica no es una mirada desinteresada ni neutra, sino sesgada y parcial. Es una mirada «construida» desde la Psicología - y más precisamente desde una cierta concepción de la Psicología - y como tal debe entenderse. Sirva ello como aviso para las páginas que siguen.

Tal vez, las múltiples caras que nos ofrecen las relaciones entre la Psicología y la Didáctica no se deban solamente a esas miradas cruzadas y diversas, que dependen tanto de nuestro punto de vista como del área de conocimiento que estemos analizando, sino también en buena medida a que esas relaciones pueden diferir según el terreno o ámbito en el que las analicemos. Como mínimo podemos diferenciar tres contextos en los que las aportaciones de la Psicología y la Didáctica pueden o no converger y que pueden representar tres formas distintas de reflexionar sobre estas relaciones mutuas y cruzadas.

En primer lugar podemos establecer esas relaciones en el terreno docente propiamente dicho, es decir, en el contexto de las actividades de aula; en este sentido cabría preguntarse cuál es la influencia respectiva que Psicología y Didáctica tienen en la labor docente real y cómo las aportaciones de una y otra pueden traducirse en una mejora efectiva de esa labor. Suele ser común, en un análisis de este tipo, partir del supuesto de que la Didáctica y la práctica docente vienen a constituir una misma realidad, siendo la Psicología el personaje invitado o visitante. Se supone que lo que el profesor hace es una labor didáctica que, en el mejor de los casos, se vería beneficiada con una adecuada aportación de la Psicología. Docente y didacta serían hasta cierto punto sinónimos. Sin embargo, una posición de este tipo - que nos tememos es muy común-implica una confusión que tiene como primera consecuencia poner en duda la propia entidad científica y epistemológica de las Didácticas Específicas, tan reclamada por éstas en los últimos tiempos. Si la historia de las Didácticas es relativamente reciente, costosa y novedosa, no puede decirse lo mismo de la docencia. $\mathrm{Ni}$ todo docente es un didacta, ni, desgraciadamente, todo didacta es siempre un docente. Es cierto que los profesores hacen una labor didáctica, pero también lo es que realizan - y no necesariamente en menor medida - una labor psicológica. Sin embargo, en muchos casos la labor didáctica y psicológica no procede de las aportaciones de la Didáctica y de la Psicología como disciplinas científicas, sino de los conocimientos intuitivos o informales que los profesores tienen sobre ellas. Es de hecho una necesidad establecer una relación más próxima entre las disciplinas científicas o académicas y las prácticas docentes a las que deberían vincularse. Eso nos lleva a un segundo nivel de análisis.

Cabe plantearse también las relaciones entre Psicología y Didáctica en el 
terreno de la formación docente. Sin estar desvinculada del análisis anterior, esta nueva perspectiva introduce aspectos nuevos, tanto desde el punto de vista teórico como institucional. El distinto papel que la Didáctica y la Psicología pueden desempeñar en la formación inicial o permanente, o los diversos modelos de formación docente serían cuestiones relevantes si analizáramos las relaciones desde esta perspectiva (por ej., Calderhead, 1988). Igualmente nos enfrentaríamos con las dificultades administrativas e institucionales que se derivan de cada una de las posiciones teóricas mantenidas, especialmente notorias en los momentos actuales. Una ventaja de este nivel de análisis es que es precisamente en el área o segmento de Formación Docente donde han encontrado acomodo buena parte de las personas que se ocupan de fundamentar y desarrollar las aportaciones didácticas. Sin embargo, no sucede lo mismo con la aportación de los psicólogos que suele hacerse desde ámbitos distintos a la formación. Las razones de esta diferencia posiblemente nos llevarían lejos del objetivo de nuestras reflexiones.

Por ello, es conveniente profundizar aún más en esas relaciones, buscando una tercera área o dominio de encuentro, que resulta en nuestra opinión más fructífero para una reflexión como la presente, como es la investigación. Si algo ha cambiado en los sucesivos encuentros - y desencuentros- entre Didáctica y Psicología es el grado en que unos y otros nos enfrentamos a problemas comunes, hablamos un lenguaje común y buscamos métodos similares para resolverlos. En definitiva, la existencia o no de un corpus de reflexión teórico común está vinculada al tipo de investigación didáctica y psicológica que sobre el aprendizaje y la enseñanza de las ciencias se viene realizando. Aquí, por razones de pertinencia y de eficacia, optaremos por analizar las relaciones entre Psicología y Didáctica en el ámbito de la investigación, ya que ello nos permitirá no sólo entender el estado actual de los conocimientos en una y otra disciplina, sino también tener una cierta prospectiva del futuro de esta extraña pareja que nos ocupa.

\section{LA INVESTIGACION DIDACTICA Y PSICOLOGICA SOBRE EL APRENDIZAJE Y LA ENSEÑANZA DE LAS CIENCIAS: CONVERGENCIAS Y DIVERGENCIAS}

En los últimos veinte años se ha asistido a una verdadera explosión de la investigación sobre el aprendizaje y la enseñanza de las Ciencias de la Naturaleza. Este incremento casi exponencial se refleja en la abundancia de Congresos, Seminarios, Sociedades Científicas y publicaciones periódicas dedicadas específicamente a la enseñanza de las ciencias. Sin duda hay muchas razones de diversa índole para este renovado interés por la educación científica, desde las necesidades derivadas por la implantación de nuevas tecnologías a la propia renovación de sistemas educativos en crisis generalizada o la propia crisis específica de la educación científica en los países desarrollados, reflejada en numerosas encuestas y estudios internacionales'.

Pero además de estas razones, hay motivos intrínsecos al propio desarrollo de una investigación didáctica específica en el área de Ciencias de la Naturaleza. La mayor parte de la investigación reciente sobre el aprendizaje y la enseñanza de las ciencias tiene un origen más didáctico que psicológico. Ello está 
sin duda relacionado con la orientación generalizada hacia lo específico que se ha producido en el mundo de la Didáctica y supone un cambio importante con respecto a la tradición que se venía manteniendo en la investigación. De hecho, si la investigación didáctica en esta área es relativamente reciente, no sucede lo mismo con la investigación psicológica. Desde hace mucho tiempo ha existido en psicología un interés por analizar la naturaleza del pensamiento científico. Podemos remontarnos a los análisis de Wertheimer (1945) sobre los procesos psicológicos implicados en los descubrimientos científicos de Einstein o Galileo, o a la distinción de Vygotsky (1934) entre la naturaleza psicológica de los conceptos científicos y los pseudoconceptos, aunque sin duda la aportación más original, influyente y decisiva es la ingente obra de Piaget, cuya Epistemología Genética ha proporcionado y sigue proporcionando hoy numerosas guías e ideas para una mejor comprensión del pensamiento científico y de los obstáculos que existen para su adquisición. Ejemplos más recientes de investigaciones psicológicas relevantes para la educación científica serían las aportaciones de Eleanor Rosch $(1978,1983)$ a la comprensión de la naturaleza del conocimiento cotidiano o «natural» o, sobre todo, los recientes desarrollos de la psicología cognitiva de la instrucción, basados en la comparación entre sujetos expertos y novatos en diversas áreas de la ciencia o el saber (Chi, Glaser y Farr, 1988; Ericsson y Smith, 1991), o incluso las aportaciones de la propia ciencia cognitiva, más orientada al diseño de sistemas tecnológicos que ayuden o faciliten el desarrollo del conocimiento científico (por ej., Langley et al., 1987).

Estos y otros muchos ejemplos de la investigación psicológica en torno al conocimiento científico y su adquisición ponen de manifiesto uno de los rasgos característicos de la misma: con ciertas excepciones, el objetivo de la mayor parte de las investigaciones es conocer mejor los procesos psicológicos, fundamentalmente cognitivos, que subyacen al pensamiento y a la solución de problemas por parte de un científico o de un experto. El acercamiento a los procesos de aprendizaje/enseñanza suele ser más bien ocasional y secundario y sólo en la medida en que es necesario para alcanzar ese objetivo primordial. En ello difieren claramente de las investigaciones didácticas, que nacen y crecen en el aula. De esta forma, como veremos más adelante, las variables y los aspectos relevantes estudiados desde una y otra disciplina no siempre son coincidentes. De hecho, los déficits más significativos de cada tipo de investigación - teórico en el caso de la Didáctica y práctico en el de la Psicología - serían deudores de su propio origen.

Por ahora nos interesa resaltar que ambos acercamientos -el psicológico y el didáctico- son divergentes no sólo en su tradición histórica, sino también en sus objetivos y enfoques dominantes en el presente ${ }^{2}$. Pero esta divergencia no es óbice para que se produzca también una convergencia entre ambos, que viene delimitada por el propio objeto de estudio de unas y otras investigaciones. Aunque difieran en su acercamiento, las investigaciones psicológicas y didácticas están empezando a encontrarse sobre un mismo objeto. Recurriendo a la terminología de Bachelard, podríamos decir que la Psicología y la Didácti$\mathrm{ca}$, aunque se interesan por aspectos distintos, acaban por tropezar con los mismos «obstáculos epistemológicos», los que se derivan de la propia naturaleza del conocimiento científico y su adquisición. De esta forma, aunque no los busquen directamente, acaban por tener problemas comunes.

Tal vez la demostración más clara de esta ambivalente relación de conver- 
gencia y divergencia simultánea lo encontremos en la contraposición de la investigación reciente desde cada una de estas perspectivas. Como se sabe, la investigación didáctica reciente ha estado dominada esencialmente por lo que podríamos llamar el enfoque de las "concepciones alternativas» o «ciencia del alumno». Con la asunción del constructivismo, el objẹto principal de esa investigación ha sido identificar las ideas que sobre la ciencia tienen los alumnos. En la década de los ochenta hemos asistido a un verdadero bombardeo de estudios sobre las ideas de los alumnos en las más diversas áreas del conocimiento científico (existen útiles «catálogos» de esas ideas como los de Driver, Guesne y Tiberghien, 1985, o Hierrezuelo y Montero, 1989). Frente a este enfoque, la investigación psicológica se ha centrado más bien en el estudio de los procesos cognitivos implicados en el pensamiento y la resolución de problemas científicos en contextos de instrucción, generalmente comparando la actuación de sujetos con distinto grado de instrucción, expertos y novatos en dominios científicos concretos (también hay algún catálogo, aunque menos exhaustivo, como los de Chi, Glaser y Farr, 1988, o Ericsson y Smith, 1991). Entre uno y otro enfoque hay significativas divergencias de partida:

(1) La investigación didáctica centra su atención en las dificultades conceptuales de los alumnos/novatos; a la investigación psicológica lo que parece interesarle realmente es el experto, aunque como metodología se realicen comparaciones con sujetos menos instruidos.

(2) Los estudios sobre concepciones alternativas toman normalmente grupos completos en situaciones muy similares a las del aula, haciendo prevalecer la validez a la fiabilidad; en cambio, las investigaciones psicológicas optan más por una metodología experimental o cuasiexperimental, en las que hay un mayor control de las variables intervinientes, con lo que se incrementa la fiabilidad en perjuicio de la validez.

(3) La investigación didáctica suele ocuparse de contenidos científicos que constituyen elementos centrales - generalmente conceptuales - del currículo; en los estudios sobre expertos y novatos el contenido de las tareas suele elegirse con criterios de conveniencia, más que de relevancia (Alexander y Judy, 1988).

(4) Los estudios didácticos están casi exclusivamente centrados en los contenidos conceptuales específicos de las Ciencias; los estudios instruccionales, aunque, como señalan autores tan significativos como Lesgold y Glaser (1989), conceden cada vez más importancia a los contenidos específicos, siguen manteniendo un mayor interés por los aspectos más generales del funcionamiento cognitivo y, como tal, dedican mayor atención a los procesos que los estudios didácticos; no sólo interesa qué ideas tienen el experto o el novato sino sobre todo cómo las utilizan en el contexto de un problema.

(5) Las investigaciones sobre concepciones alternativas se han centrado muchas veces en una sola idea o concepto aislado, identificando nociones precientíficas, mientras que los estudios psicológicos tienden a mostrar un mayor interés por la estructura y organización de los conocimientos en la mente de los alumnos, evitando analizarlos como contenidos individuales (Carey, 1985; Chi, Glaser y Rees, 1982).

Pero, junto a estas divergencias de partida en el acercamiento al conocimiento científico y su adquisición, encontramos también significativas convergencias en los resultados obtenidos desde ambos enfoques, que pueden concentrarse en dos paralelismos fundamentales: 
(1) Las investigaciones psicológicas sobre el pensamiento y la resolución de problemas han ido abandonando cada vez más la idea de que las personas nos basamos en una racionalidad lógica y científica; en vez de ello nos enfrentamos a los problemas e intentamos resolverlos guiados por sesgos y heurísticos ajenos a las formas lógicas usuales (por ej., Carretero y García Madruga, 1984; Kahneman, Tversky y Slovic, 1984; Pérez Echeverría, 1990; Sternberg y Frensch, 1991). Muchos de estos sesgos o modos informales de resolver problemas se han identificado no sólo en el pensamiento cotidiano o en el de sujetos novatos resolviendo problemas científicos (por ej., Kuhn, 1991; Kuhn, Amsel y O'Loughlin, 1988; Sternberg y Frensch, 1991), sino también, aunque sea en menor medida, en la solución de problemas por expertos o incluso científicos renombrados (Tweney, Doherty y Mynatt, 1981) reaparecen de modo sistemático en la literatura didáctica como ejemplos de errores que los alumnos cometen en la resolución de problemas en el aula (por ej., Driver, 1983, Wellington, 1989, o el excelente retrato que Claxton, 1991, hace de los trabajos de laboratorio de los alumnos), mostrando la existencia de un pensamiento cotidiano o informal bien diferenciado de lo que suele entenderse por pensamiento científico y que persiste no sólo en los alumnos sino en el razonamiento cotidiano de todos nosotros (por ej., Holland et al., 1986; Kuhn, 1991).

(2) Las «concepciones alternativas» que la investigación didáctica ha mostrado que los alumnos mantienen con respecto a los más diversos fenómenos científicos (por ej., Driver, Guesne y Tiberghien, 1985; Hierrezuelo y Montero, 1989; Lucas y Black, en prensa; Osborne y Freyberg, 1985) reaparecen a su vez en los estudios instruccionales que utilizan sujetos adultos resolviendo problemas en dominios científicos ajenos a su experiencia (Pozo y Carretero, 1992). La similitud no alcanza sólo a que alumnos adolescentes y adultos novatos tengan las mismas concepciones alternativas sobre los fenómenos científicos, sino que incluso las características genéricamente atribuidas a las ideas espontáneas de los alumnos sobre la ciencia desde la investigación didáctica (por ej., Andersson, 1986; Driver, Guesne y Tiberghien, 1985) son muy similares a las encontradas en la estructura conceptual del conocimiento de los sujetos novatos en la tradición de investigación psicológica (por ej., Chi, Glaser y Rees, 1982; para una síntesis entre ambas véase Pozo y Carretero, 1987; Pozo et al., 1991b).

En definitiva, las investigaciones didácticas y psicológicas sobre el aprendizaje de las ciencias se están encontrando con datos o problemas similares aun cuando sus puntos de partida, tanto teóricos como metodológicos, son en muchos casos muy divergentes. Podríamos decir que, al menos en el área de investigación que nos ocupa, la Didáctica y la Psicología son como dos trenes circulando en paralelo pero en direcciones opuestas. Uno, el de la Didáctica, procede del conocimiento disciplinar específico y se va viendo obligado a situar ese conocimiento disciplinar en contextos más generales, tanto en una dimensión curricular como meramente psicológica, mientras que el otro, el de la Psicología, ha optado siempre por los aspectos generales del conocimiento y sólo recientemente, y de un modo aún tímido, ha comenzado a descubrir la relevancia de los conocimientos disciplinares específicos. Es ahí precisamente, en los contenidos y en su construcción en la mente de los alumnos, donde el encuentro de esos dos trenes parece hoy inevitable. Lo que nos acerca a unos y a otros es el propio objeto de estudio; aunque no lo queramos y a veces no lo creamos, estamos estudiando los mismos problemas desde perspectivas distintas y potencialmente complementarias. 
Sin embargo, este acercamiento sobre el mismo objeto de estudio es relativamente reciente y tiene mucho que ver con el propio desarrollo interno de la Didáctica de las Ciencias por un lado y de la Psicología por otro, que les han llevado a coincidir en una misma concepción de los procesos de aprendizaje/enseñanza, caracterizada por el constructivismo, que ha hecho posible que esas miradas cruzadas llegaran a encontrarse.

Como señalábamos al comienzo del artículo, este encuentro reciente está condicionado, en el área de Ciencias de la Naturaleza, por un incremento exponencial de la investigación didáctica en los últimos años. El paraguas del constructivismo ha impulsado una gran cantidad de investigación sobre las ideas y, en menor medida, las destrezas que los alumnos ponen en acción ante una tarea científica. En cambio, la investigación psicológica sobre el aprendizaje y la enseñanza de las ciencias no ha crecido en los últimos años, sino más bien al contrario. Si la década de los setenta fue para la enseñanza de la ciencia la «edad de Piaget», la recién terminada década de los ochenta puede calificarse muy bien como la «época de las concepciones alternativas». La insatisfacción con el concepto piagetiano de estadio (Driver y Easley, 1978; Gilbert y Swift, 1985), unida a otros varios factores psicológicos y didácticos, ha hecho que las investigaciones se hayan ido orientando progresivamente hacia el estudio de las ideas de los alumnos sobre fenómenos científicos específicos.

Como la investigación psicológica ha estado en gran medida apoyada en una creencia en procesos y capacidades generales, la aportación psicológica a la investigación en dominios específicos es y ha sido bastante escasa. Aunque hay una creciente consciencia de la importancia del contenido al que se aplican los procesos (por ej., Alexander y Judy, 1988; Lesgold y Glaser, 1989), las concepciones predominantes en psicología siguen siendo «generalistas». Como máximo se asume una posición «débil» con respecto a la influencia del contenido, según la cual éste influiría en la facilidad o disponibilidad de aplicación de un proceso o de un conocimiento a un dominio dado. Son muy pocos, por el contrario, los que ponen el acento en el contenido y se defienden, como Ceci y Nightingale (1990, p. 40), que «cada proceso está inicialmente vinculado a un dominio particular». Esta última concepción requeriría una «Psicología de los Contenidos» - que se traduciría en Psicologías Específicas- además de la «Psicología de los Procesos» -o Psicología General- al uso.

Como consecuencia de lo anterior, tras la crisis del modelo generalista y las insuficiencias demostradas por el procesamiento de información para abordar los contenidos de los procesos mentales (Pozo, 1989), la investigación psicológica está carente de un modelo integrador que le permita abordar los problemas del contenido. Así, la investigación psicológica reciente sobre la comprensión y el aprendizaje de la ciencia, aunque predominantemente instruccional, resulta difícil de analizar de modo sucinto, dada la diversidad de planteamientos teóricos subyacentes a lo que Rivière (1987) denomina la ya vaga etiqueta de lo cognitivo. En cambio, la investigación didáctica, siendo más abundante, ha estado más guiada por demandas prácticas que por consideraciones teóricas. Recurriendo a la metáfora anterior, podríamos decir que los trenes de la Didáctica y la Psicología son gravemente desiguales. La investigación didáctica es un tren con una máquina y muchísimos vagones, mientras que en la investigación psicológica parece haber más máquinas que vagones y no siempre tirando en la misma dirección. Dado que posiblemente entre los lectores haya más viajeros interesados 
en subirse al tren de la Didáctica, creemos más eficaz para nuestros objetivos dedicar un esfuerzo a analizar críticamente, desde planteamientos psicológicos, el tumultuoso viaje de la investigación didáctica, para al final del trabajo recuperar unas consideraciones más generales sobre las relaciones entre ambos tipos de investigación.

\section{MAS ALLA DE LAS CONCEPCIONES ALTERNATIVAS: UN ANALISIS CRITICO DE LA INVESTIGACION DIDACTICA RECIENTE}

A pesar de su aceptación generalizada entre los investigadores e incluso, de modo creciente, entre los profesores, existen algunos rasgos, tanto empíricos como teóricos, de la investigación didáctica basada en el enfoque de las concepciones alternativas que merecen una reflexión crítica. Resulta significativa la escasez de críticas que este enfoque ha recibido, tanto por sus detractores como por sus propios defensores, ya que ello no se debe tanto a la propia consistencia teórica y experimental del enfoque cuanto a una aceptación acrítica de muchos de sus supuestos, que reduce la investigación a una acumulación de datos descriptivos sobre las concepciones alternativas de grupos determinados de alumnos en tareas determinadas, a partir de las cuales, sin apenas restricciones, se establecen generalizaciones susceptibles de afectar a la propia estructura del currículo. Merece por tanto la pena esforzarse en determinar las verdaderas aportaciones empíricas y teóricas realizadas por este enfoque y las cautelas con que deben ser aceptadas.

Sin duda lo que más ha favorecido la rápida aceptación del enfoque de las concepciones alternativas ha sido la abundancia de datos mostrando que en las más diversas áreas y tareas los alumnos disponen de ideas previas que utilizan para dar significado a los hechos. Este verdadero alud de datos requería una concepción constructivista del conocimiento y un abandono de la idea asociacionista del aprendizaje que subyacía a ciertos modelos de enseñanza, lo que ha supuesto un acercamiento de la Didáctica de las Ciencias a los planteamientos de la psicología cognitiva del aprendizaje (Pozo, 1989).

No obstante la apabullante cantidad de datos acumulados en la última déca$\mathrm{da}$, algunos autores han puesto en duda su relevancia o la interpretación que habitualmente se hace de los mismos. Con respecto a lo que podemos llamar críticas metodológicas, se pueden clasificar a partir de los dos criterios esenciales que debe reunir toda investigación: validez y fiabilidad.

Suele decirse que los datos recogidos en una investigación son válidos cuando «miden lo que dicen medir». Algunos autores han puesto en duda que algunas o muchas de las situaciones experimentales en que se dice estar estudiando las concepciones alternativas de los alumnos midan realmente las ideas previas de los alumnos y no otra serie de factores contextuales o situacionales de menor relevancia para la enseñanza de la ciencia (falta de atención, tareas engañosas o paradójicas, respuestas sugeridas, etc.). La propia Rosalind Driver (1988) ha advertido contra la tendencia excesiva a creer que toda respuesta dada por los alumnos en una situación de este tipo refleja verdaderamente una concepción alternativa.

Lo cierto es que más allá de ciertas cautelas y criterios que están comenzando a desarrollarse (por ej., Giordan y De Vecchi, 1987) ha habido una tendencia 
a aceptar como válido cualquier dato, sin exigir ningún rigor metodológico. Como señalan Engel Clough y Driver (1986) el desprecio de este enfoque con respecto a las variables contextuales sólo es comparable al del propio Piaget. La diferencia es que Piaget renunciaba a estudiar estas variables por consideraciones teóricas - le interesaba el sujeto «epistémico", no el alumno real-mientras que a este enfoque esas mismas consideraciones deberían llevarle a analizar con mayor cautela todos los factores contextuales que afectan al rendimiento de los alumnos en una tarea determinada, y con ellos a manipular y controlar las variables del sujeto y de la tarea que pueden afectar a ese rendimiento.

La creencia firmemente asentada en la estabilidad y «validez» de las concepciones alternativas se desvanece en cuanto se varían mínimamente las instrucciones de presentación de una tarea. Yates et al. (1988) han comprobado que en un área tan estudiada como la mecánica newtoniana la simple modificación del enunciado de un ítem puede variar los resultados obtenidos. Un más riguroso y controlado análisis de las variables que afectan a las respuestas de los alumnos conduciría a este enfoque a una investigación que permitiera elaboraciones teóricas que fueran más allá de lo simplemente descriptivo. La aceptación acrítica de ciertos modelos de representación conceptual, que como veremos más adelante no son compartidos por la psicología cognitiva actual, podrá superarse en la medida en que el método descriptivo sea progresivamente reemplazado por estudios experimentales en los que se controlen ciertas variables, tanto de la tarea como del alumno, teóricamente relevantes y por tanto informativas para la teoría. Enfrentado a una tarea, el alumno pone en marcha una serie de procesos psicológicos que interactúan en su rendimiento y que no siempre reflejan concepciones estables y duraderas, sino que muchas veces son el producto de la interacción de las condiciones de la tarea y del propio alumno que inciden en la activación de unos u otros procesos.

En cualquier caso, aunque no siempre estudien lo que creen estar estudiando las investigaciones descriptivas realizadas hasta la fecha tienen una gran relevancia para la enseñanza en esta área. Sin embargo, estos propios estudios descriptivos, aun asumiendo su validez, son en muchos casos criticables por su escasa fiabilidad, es decir, resultan difícilmente replicables. La metodología utilizada en estas investigaciones, tanto desde el punto de vista del muestreo y de las condiciones de aplicación, como de los criterios de análisis de respuestas y el tratamiento estadístico que reciben, no es siempre la adecuada.

La selección de las muestras suele estar bastante alejada de los criterios de aleatoriedad exigibles en la investigación en ciencias sociales. Tampoco es habitual que se diferencie a los sujetos en función de ciertas variables potencialmente moduladoras. Aunque puede esperarse razonablemente que los resultados obtenidos con uno o unos pocos grupos de alumnos - muchas veces clases enteras compuestas de alumnos del profesor/investigador - sean representativos del total de la población de adolescentes, serían precisas mayores cautelas en cuanto al control de la edad, nivel de conocimientos previos y otras variables individuales cuyo control no sólo incrementaría la fiabilidad de estos estudios sino también su interés teórico. Se trataría de identificar las variables que diferencian a unos alumnos de otros en lugar de establecer, como hasta ahora, un retrato ideal del alumno.

También dificulta la interpretación de estos estudios la escasez de análisis estadísticos realizados y, en algunos casos, la poca claridad de los criterios o 
niveles de respuesta utilizados. La mayor parte de los estudios se limitan a presentar porcentajes, sin aderezarlos siquiera con un análisis estadístico con respecto a las diferencias halladas. De hecho, no todas las concepciones alternativas surgen con la misma fuerza o si se quiere con la misma fiabilidad. Sería preciso extremar el rigor en este aspecto, ya que sólo así podremos atribuir significado a las diferencias observadas.

Lamentablemente es menor aún el número de trabajos que establecen manipulaciones experimentales de variables potencialmente relevantes. Tampoco se recurre al análisis correlacional, que a pesar de sus limitaciones, puede proporcionar información sobre el grado de consistencia y homogeneidad de las diversas concepciones utilizadas por los alumnos $y$, con ello, ofrecernos datos sobre uno de los aspectos esenciales en la comprensión de la ciencia: la organización que adoptan las ideas científicas en la mente de los alumnos.

En definitiva, hay varias objeciones metodológicas que, sin menoscabar la relevancia de los datos obtenidos sobre las concepciones alternativas de los alumnos, deben tenerse en cuenta antes de atribuirles un significado teórico. Aunque los datos, recogidos en países con alumnos y contextos educativos distintos, son tan generosos como indiscutibles, su interpretación ya es más discutible en la medida en que, si asumimos nuestra propia epistemología constructivista, depende del marco teórico asumido por cada cual. Desde el punto de vista de la teoría cabe también hacer algunas reflexiones críticas con respecto al estatuto conceptual de las concepciones alternativas.

El enfoque de las concepciones alternativas ha supuesto, por oposición al piagetiano, un énfasis mayor en los conocimientos específicos que en las estructuras cognitivas generales. Ello ha traído consigo una cierta «desintegración» del alumno, que ha pasado de poseer un sistema cognitivo organizado y predecible a disponer de un número no determinado de concepciones poco conectadas entre sí y por tanto difícilmente predecibles. Esta evolución, que como ya se ha señalado es paralela en algún sentido a la de la propia psicología cognitiva, supone que los modelos explicativos suelen estar centrados en las tareas más que en el sujeto, lo que justifica la adopción de criterios disciplinares o específicos en la organización del currículo.

Esta tendencia «modular» en los modelos propuestos para explicar cómo entienden la ciencia los alumnos está justificada en parte en los datos que ponen en duda la existencia de estadios o estructuras cognitivas homogéneas en el pensamiento de los alumnos. Sin embargo, parece que el conocimiento científico de los alumnos no es tan homogéneo como Piaget suponía, pero tampoco resulta tan heterogéneo o dispar como el enfoque de las concepciones alternativas supone actualmente. Aunque no se han hallado correlaciones entre tareas que según Piaget debían estar altamente relacionadas, se han observado correlaciones estadísticamente significativas entre tareas formales que desde el punto de vista de las concepciones alternativas no serían predecibles (por ej., Demetriou, Efklides y Gustaffson, en prensa; Lawson, 1977, 1989; entre nosotros, por ej., López Rupérez y Palacios, 1988). Los pocos trabajos que han intentado establecer relaciones entre las concepciones alternativas de los alumnos en diversas tareas han observado que éstas no son completamente independientes, ya que tienen un nivel de coherencia variable, pero tampoco constituyen un sistema de conjunto único y general (por ej., Engel Clough y Driver, 1986; Gómez Crespo, Pozo y Sanz, 1992; Jiménez Aleixandre, 1990; Pozo y Carretero, 1992). Los da- 
tos parecen reclamar niveles de generalidad $\mathrm{u}$ homogeneidad intermedios a los previstos por Piaget o las concepciones alternativas (Case, 1991, 1992).

El problema de la falta de organización cognitiva del alumno en la investigación didáctica está muy relacionado con el propio concepto de las «concepciones alternativas» o «ideas previas» de los alumnos. De hecho, el enfoque de las concepciones alternativas se caracteriza por una aceptación acrítica de algunos de los postulados y conceptos de la modema psicología cognitiva, importando de ella términos y conceptos (constructivismo, esquema, metaconocimiento, zona de desarrollo potencial, etc.) que la propia psicología cognitiva no ha conseguido aún aclarar suficientemente o con respecto a los cuáles existen posiciones encontradas o incompatibles. Esta importación acrítica, que es útil en la medida en que ayuda a comprender mejor a los alumnos, plantea a medio plazo problemas conceptuales en la investigación, ya que impiden un análisis más pormenorizado de las dificultades de aprendizaje de los alumnos, debido a la propia vaguedad de los conceptos empleados.

Desde el punto de vista de la psicología cognitiva actual, el propio concepto de concepción alternativa se hallaría muy próximo a muchos de sus constructos teóricos, pero sin que sea posible, dada su indefinición ${ }^{3}$, hacerlo coincidir en ninguno de ellos, por lo que resulta muy difícil abordar, desde una perspectiva psicológica, la representación que tienen los sujetos de los fenómenos científicos, sus «concepciones alternativas». La naturaleza y el funcionamiento de las representaciones constituyen un problema crucial, no sólo desde un punto de vista psicológico sino también didáctico. Sin embargo, con algunas excepciones (por ej., Giordan y De Vecchi, 1987), es una cuestión que apenas ha sido abordada y respondida desde este enfoque.

Mientras que las concepciones alternativas se sitúan en el nivel de análisis del conocimiento intuitivo de las personas —es decir, lo que esas personas hacen o dicen con respecto a una situación concreta- el nivel representacional debería referirse más bien a las estructuras y procesos que subyacen a esas concepciones y que las bacen posibles. La distinción entre estos dos niveles, que es básica en psicología cognitiva (Rivière, 1987), no se establece al hablar de concepciones alternativas, por lo que ambos se confunden y se atribuyen en ocasiones propiedades del uno al otro. Al igual que los alumnos atribuyen propiedades observables o macroscópicas a la estructura no observable de la materia, la confusión entre la conducta observable y las representaciones que están en su origen puede conducir a atribuir a esas representaciones científicas del alumno propiedades de las que en realidad carecen.

Desde nuestro punto de vista, el concepto de «concepto» habitualmente usado en este enfoque sería más preciso si se incorporaran dos distinciones fundamentales establecidas por las modernas teorías de la representación y el aprendizaje cognitivo. En primer lugar, las concepciones alternativas se ajustan más a lo que hoy día se conoce como una categoría natural -o concepto probabilístico de límites difusos - que a una categoría lógica, bien definida, como son los conceptos científicos. Esto implicaría que el cambio conceptual alentado por este tipo de modelos (por ej., Haswhew, 1986; Hewson y Hewson, 1984; Posner et al., 1982) no supone sólo el cambio de un concepto por otro sino sobre todo el cambio de una forma de conceptualizar - la categoría natural - por otra - el concepto científico.

Además, las concepciones alternativas, lejos de ser conceptos estables y du- 
raderos, presentes explícitamente en la memoria de los alumnos, serían representaciones implícitas, que en muchos casos el alumno construiría ad boc o sobre la marcha para atender a las demandas de una tarea (por ej., Holland et al., 1986; Pozo et al., 1991a, 1992; Rodrigo, 1985). La atribución de este carácter implícito al conocimiento espontáneo es uno de los rasgos más sobresalientes de los estudios actuales sobre las representaciones que usamos las personas, concediendo especial atención a los factores o variables contextuales que determinan la activación de una u otra representación en un momento dado. En otras palabras, no se trataría tanto de investigar qué concepción tienen los alumnos sobre un fenómeno determinado cuanto de analizar cuál de sus representaciones implícitas activarán ante una tarea y qué variables determinan esa activación. Desde esta perspectiva el cambio conceptual implicará no sólo facilitar la construcción de nuevas representaciones sino proporcionarles indicios para su activación selectiva, de forma que los alumnos no sólo tengan conocimientos científicos sino que además sepan usarlos en el momento adecuado.

Esta distinción está ausente en la investigación de las concepciones alternativas y sin embargo podría ayudar a comprender algunos de los «errores» que cometen los alumnos. Para ello, como señalan Engel Clough y Driver (1986) hay que profundizar en el uso que los alumnos hacen de sus concepciones $y$, lo que es más importante, adoptar una perspectiva teórica en el análisis de las concepciones de los alumnos que integrase algunos de los aspectos que venimos señalando.

En nuestra opinión, la investigación psicológica sobre el aprendizaje y la enseñanza de la ciencia puede ayudar a interpretar de modo más complejo las relaciones entre el conocimiento implícito o intuitivo de los alumnos y las actividades de aprendizaje y enseñanza que se realizan en el aula. Sin embargo, para que esta ayuda sea más eficaz es necesario que la propia investigación psicológica sobre el aprendizaje de las ciencias se apoye a su vez en la investigación didáctica. Como hemos señalado anteriormente, la investigación psicológica necesita profundizar en los contenidos del conocimiento y la forma en que se adquieren en contextos de instrucción formal $e$ informal. En otras palabras, el constructivismo ha venido a crear una relación, no siempre deseada o bien aceptada, de mutua dependencia, que idealmente, debería adoptar formas de cooperación. Más allá de la posibilidad, nada desdeñable, de encontrar respuestas «didácticas» a las preguntas planteadas por la investigación «psicológica», y viceversa, existen algunos aspectos o problemas comunes a ambos tipos de investigación, derivados de los análisis anteriores, que pueden constituir de algún modo una agenda de trabajo común.

\section{HACIA UNA AGENDA COMUN: DOS PROBLEMAS PENDIENTES E INTERDEPENDIENTES}

En un apartado anterior, destacábamos lo que a nuestro entender constituyen algunas divergencias entre los planteamientos de la investigación psicológica y didáctica, que sin embargo no impiden la aparición de algunos resultados muy similares en ambas tradiciones. Pero junto a estos datos o resultados comunes o convergentes, encontrados desde ambos tipos de investigación, hay también unos rasgos deficitarios comunes, es decir, carencias que pueden observarse 
de modo similar en los estudios didácticos y psicológicos. La investigación psicológica y didáctica tienen aún sin resolver en su agenda como mínimo dos problemas que, aunque originados en tradiciones distintas, han resultado ser comunes. Nos estamos refiriendo a los dos problemas interrelacionados de (1) la influencia de las variables contextuales en el uso del conocimiento y (2) del aprendizaje concebido como cambio conceptual.

Sin duda, no es aventurado afirmar que tanto la investigación didáctica como la psicológica han tenido escasamente en cuenta hasta la fecha las variables contextuales. Sin embargo, desde uno y otro campo está habiendo un interés creciente por considerar los conocimientos científicos de los alumnos en su contexto de uso (Driver, 1989; Di Sessa, 1988). Los recientes desarrollos psicológicos sobre las representaciones o teorías implícitas que compiten por ser activadas en la mente de los sujetos (por ej., Holland et al., 1986; Pozo et al., 1992; Rodrigo, 1985) pueden ofrecer un marco de análisis, siempre y cuando se acompañen de investigaciones que identifiquen las variables que facilitan en cada contexto la activación de unos conocimientos $o$ ideas $u$ otros. Esta tendencia es coincidente con el creciente interés por el «aprendizaje en contexto» (o situated learning), que puede observarse en muchas otras áreas de la investigación educativa, que están comenzando a reclamar un análisis del conocimiento de los alumnos en sus contextos de uso tanto dentro del aula como en situaciones no escolares (Lave y Wegner, 1991; Resnick, 1989).

Obviamente, en el aula uno de los factores más relevantes es precisamente la interacción entre profesor y alumnos en el contexto de las metas fijadas por las actividades de aprendizaje y enseñanza. Dado que estas metas escolares generalmente difieren de las metas existentes en la actividad cotidiana, reduciendo la probabilidad de activación del conocimiento cientifico fuera del aula. Como Claxton (1991) ha mostrado recientemente, las actividades de aprendizaje/enseñanza en las aulas suelen organizarse de forma muy diferente a cualquier otro contexto de la vida real, por lo que muchas veces resulta difícil, cuando no imposible, establecer un «puente» a través del cual transferir el conocimiento de un dominio a otro. En este sentido, la investigación no sólo debería ocuparse de los contextos de adquisición del conocimiento científico en el aula -esencialmente regidos por las demandas mutuas generadas por la interacción entre profesor y alumnos en el marco de un contenido curricular concreto (por ej., Coll y Solé, 1990) - sino también considerar los contextos de uso del conocimiento científico más allá del aula, es decir, la supuesta utilidad del conocimiento científico para la comprensión y la resolución de problemas en la vida cotidiana (Claxton, 1991). En todo caso, para que la Psicología pueda hacer una aportación sistemática a este «aprendizaje en contexto", tanto mediante un análisis de las interacciones escolares como de las situaciones cotidianas no escolares, es preciso que incorpore desde dentro de sus modelos un análisis de los contenidos y que no se limite a un discurso nuevamente generalista sobre el formato de esos contextos, con independencia del contenido al que se refieran.

Una de las implicaciones de los modelos contextuales es que, manteniéndose dentro de los supuestos constructivistas, deben conllevar una nueva concepción del cambio y del aprendizaje, lo cual nos conduce al segundo de los problemas no resueltos hasta ahora ni por la Didáctica ni por la Psicología, como es el problema del aprendizaje concebido como un cambio, ya sea conceptual, procedimental y/o actitudinal. 
Más allá de posiciones teóricas a priori, tanto las investigaciones didácticas como las psicológicas tropiezan con dificultades empíricas para establecer la dinámica de la adquisición y el cambio de conocimiento. Generalmente, unas y otras investigaciones recurren al establecimiento de niveles de respuesta ante una tarea, jerárquicamente ordenados, pero sin lograr explicar o justificar plenamente de qué modo los sujetos van superando o ascendiendo por esa jerarquía. Desde ambos enfoques se alude al «cambio conceptual» (Carey, 1985; Hashweh, 1986; Hewson y Hewson, 1987; Jiménez Aleixandre, 1990; Posner et al., 1982; Pozo, 1989; West y Pines, 1985) y «metodológico» (Gil y Carrascosa, 1985), a la conveniencia de promover el conflicto cognitivo o sociocognitivo como motor del cambio y del aprendizaje (por ej., Brown y Palincsar, 1989; Giordan y De Vecchi, 1987).

Sin embargo, las investigaciones, sean didácticas o psicológicas, sobre el papel del conflicto en el aprendizaje de las ciencias está lejos de ofrecer datos claros y concluyentes, aunque se apunten algunas tendencias que, frente a ciertas interpretaciones simples o lineales, subrayan que la complejidad de los procesos implicados en el cambio conceptual y/o metodológico puede dar lugar a muy diversos efectos del conflicto en función del contexto en que se produzca (Driver, 1989; Kuhn, Amsel y O'Loughlin, 1988). Tal vez, sugieren estas investigaciones, establecer una ruta universal o incluso habitual para el cambio conceptual sea muy aventurado, porque en realidad hay muchas rutas y formas de acceder a esos nuevos niveles de conocimiento conceptual o procedimental. Tal vez, nos recuerdan, los conflictos puedan tener efectos bien diferentes en función del contexto en que se produzcan. En definitiva, como señalábamos unas líneas más arriba, una concepción del aprendizaje en contexto, hacia la que parece orientarse la investigación, requiere una nueva forma de entender la construcción del conocimiento y, en definitiva, el cambio conceptual y procedimental. No se trataría sólo de adquirir conocimientos sino también, y por encima de todo, de aprender a activar esos conocimientos en los contextos adecuados. Los alumnos no abandonarian un conocimiento por otro, sino que dispondrían de conocimientos alternativos que competirian por ser activados. Las concepciones serían alternativas también dentro del alumno (Gómez Crespo, Pozo y Sanz, 1992). El cambio producido por el aprendizaje no debería entenderse como la sustitución de un conocimiento por otro más elaborado, sino más bien como un cambio en la probabilidad de activación de los conocimientos en función de ciertas variables contextuales. En una perspectiva de futuro son estas variables - que incluirían desde el modo de presentación de la tarea, la meta u objetivo de la misma, factores situacionales internos del alumno, el contexto social en que se produce, etc.los mejores candidatos para concentrar la atención de psicólogos y didactas interesados en la adquisición, pero también en el uso, de los conocimientos científicos.

\section{Notas}

${ }^{1}$ Por ejemplo, recientemente la International Association for the Evaluation of Educational Achievement (IEA, 1991, 1992) acaba de editar dos nuevos volúmenes sobre una evaluación internacional de la intrucción científica en una amplia gama de países, que viene realizándose periódicamente desde 1984 . 
${ }^{2}$ Somos muy conscientes de que, como las matriusbleas rusas cada uno de estos acercamientos -el didáctico y el psicológico- esconde en sí mismo una gran variedad de planteamientos y enfoques diferentes. En algunos sentidos, resultaría más enriquecedor profundizar en las diferencias entre las diversas Psicologías y Didácticas, pero «por exigencias del guión» nos atendremos a la sumaria comparación entre la investigación procedente de una y otra disciplina, aunque ello implique pasar por alto muchos matices.

${ }^{3}$ Las ideas o conocimientos previos de los alumnos, que aquí recogemos bajo la discutible etiqueta de «concepciones alternativas», han recibido de hecho casi tantos nombres como investigadores se han acercado a ellas. Giordan y De Vecchi (1987) afirman haber encontrado hasta 28 denominaciones distintas para referirse a las ideas de los alumnos sobre los hechos científicos.

\section{Referencias}

Alexander, P. A., y Judy, J. E. (1988). The interaction of domain-specific and strategic knowledge in academic performance. Review of Educational Research, 58, 375-404.

AnDersson, B. (1986). The experimental gestalt of causation: a common core to pupils' preconceptions in science. European Journal of Science Education, 8 (2), 155-171.

Brown, A. L., y Palinesar, A. S. (1989). Guided, cooperative learning and individual knowledge acquisition. En L. Resnick (ed.), Knowing, learning and instruction, Hillsdale, N. J.: LEA.

CalderheAd, J. (ed.) (1988). Teachers' professional leaming. Londres: Falmer Press.

Carey, S. (1985). Conceptual change in childbood. Cambridge, Mass: M. I. T. Press.

Carretero, M., y Garcia-Madruga, J. A. (Eds.) (1984). Lecturas de psicologia del pensamiento. Madrid: Alianza.

CASE, R. (ed.) (1991). The mind's staircase. Hillsdale, N. J.: LEA.

CASE, R. (1992). The role of central conceptual structures in the development of children's scientific and mathematical thought. En A. Demetriou; M. Shayer y A. Efklides (Eds.). Neopiagetian theories of cognitive development: implications and applications to education. Londres: Routledge y Kegan Paul.

Ceci, S. J., y Nightingale, M. N. (1990). The entanglement of knowledge and process in development: toward a tentative framework for understanding individual differences in intellectual development. En W. Schneider y F. E. Weinert (Eds.), Interactions among aptitudes, strategies and knowledge in cognitive performance. Nueva York: Springer-Verlag.

CHI, M. T. H.; G LASER, R., y FARR, M. (Eds.) (1988). The nature of expertise. Hillsdale, N. J.: Erlbaum.

Chi, M. T. H.; Glaser, R., y Ress, E. (1982). Expertise in problem solving. En R. J. Sternberg (Ed.), Advances in the psychology of human intelligence. Hillsdale, N. J.: LEA.

Claxton, G. (1991). Educating the inquiring mind. The challenge for school science. Londres: Harvester.

Coll, C., y Solḱ, I. (1990). La interacción profesor/alumno en el proceso de enseñanza y aprendizaje. En C. Coll, J. Palacios y A. Marchesi (eds.). Desarrollo psicológico y educación, II. Psicología y Educación. Madrid: Alianza.

Demetriou, A.; Efklides, A., y Gustafsson, J. (1992). Structural systems in developing cognition, science and education. En A. Demetriou, M. Shayer y A. Efklides (Eds.), Neopiagetian theories of cognitive development: Implications and applications. Londres: Routledge y Kegan Paul.

Di Sessa, A. (1988). Knowledge in pieces. En G. Forman y P. B. Pufall (eds.). Contructivism in the computer age. Hillsdale, N. J.: LEA.

DrIver, R. (1983). The pupil as scientist? Milton Keynes: Open University Press.

DrIVER, R. (1988). Un enfoque constructivista para el desarrollo del currículo en ciencias. Enseñanza de las Ciencias, 6 (2), 109-120.

DRIVER, R. (1989). Students' conceptions and the learning of science. International Joumal of Science Education, 11, 481-490.

Driver, R., y EASLEY, M. (1978). Pupils and paradigms: a review of literature related to concept development in adolescent science education. Studies in Science Education, 5, 61-84.

Driver, R.; Guesne, E., y Tiberghien, A. (Eds.) (1985). Children's ideas in science. Milton Keynes: Open University Press. Trad. cast. de P. Manzano: Las ideas cientificas en la infancia y la adolescencia. Madrid: Morata, 1989.

Engel Clough, E., y Driver, R. (1986). A study of consistency in the use of students' conceptual frameworks across differnr task contexts. Science Education, 70 (4), 473-496.

ERICsson, K. A., y SMTH, J. (eds.) (1991). Toward a general theory of expertise. Cambridge, Mass.: Cambridge University Press.

GIL, D., y Carrascosa, J. (1985). Science learning as a conceptual and methodological change. European Joumal of Science Education, 7 (3), 231-236. 


\section{2}

Gilbert, J. K., y Swift, D. J. (1985). Towards a lakatosian analysis of the piagetian and alternative conceptions research programs. Science Education, 69 (5), 681-696.

Giordan, A., y DE VECCHI, G. (1987). Les origines du Savoir. Delachaux et Niestlé: Neuchatel.

Gomez Crespo, M. A.; Pozo, J. I., y Sanz, A. (1992). Students' conceptions about conservation of matter (enviado para publicación).

HASHWEH, M. Z. (1986). Toward an explanation of conceptual change. European Journal of Science Education, 8 (3), 229-249.

Hewson, P. W., y Hewson, M. G. (1984). The role conceptual conflict in conceptual change and the design of science instruction. Instructional Science, 13, 1-13.

Hierrezuelo, J., y Montero, A. (1988). La ciencia de los alumnos. Barcelona: Laia/MEC.

Holland, J. M.; Holyoax, K. J.; Nisbett, R. E., y Thagard, P. R. (1986). Induction. Processes of inference, leaming and discovery. Cambridge, Mass: The MIT Press.

I. E. A. (International Association for the Evaluation of Educational Achievement) (1991). Science Acbievement in twenty three countries from 1984 to 1990. Londres: Pergamon Press.

I. E. A. (International Association for the Evaluation of Educational Achievement) (1992). Science Achievement in twenty three countries. Londres: Pergamon Press.

Jiménez AleixandRe, M. P. (1990). Los esquemas conceptuales sobre la selección natural: análisis y propuestas para un cambio conceptual. Madrid: Tesis Doctoral, facsímil, Universidad Complutense.

Kahneman, D.; Slovic, P., y Tersky, A. (Eds.) (1984). Judgement under uncertainty: beuristics and biases. Cambridge: Cambridge University Press.

Kunn, D. (1991). The skills of argument. Cambridge, Mass.: Cambridge University Press.

Kunn, D.; Amsel, E., y O'Loughlin, M. (1988). The development of scientific thinking skills. Orlando: Academic Press.

Langley, P.; Simon, H. A.; Bradshaw, G. L., y ZytKow, J. W. (1987). Scientific discovery: computational explorations of the creative processes. Cambridge, Ma.: M.I.T. Press.

Lave, J., y Wenger, E. (1991). Situated leaming. Cambridge, Mass.: Cambridge University Press.

LAwson, A. E. (1977). Relationships among performances on three formal operational tasks. The Joumal of Psycbology, 96, 235-241.

LAWrSON, A. E. (1989). Research on advanced reasoning, concept acquisition and a theory of science instruction. En P. Adey (Ed.). Adolescent development and school science. Londres: Falmer.

López Rupêrez, F., y Palacios, C. (1988). La exigencia cognitiva en física básica. Un análisis empíri. co. Madrid: MEC.

LuCAS, A., y B BACK, P. (eds.) (en prensa). Childrens' implicit ideas in science. Londres: Routledge \& Kegan Paul.

Osborne, R. J., y Freyberg, P. (1985). Learning and science: the implications of «children's science». N. Zelanda: Heinemann Educational.

Pérez Echeverría, M. P. (1990). Psicología del razonamiento probabilístico. Madrid: Servicio de Publicaciones de la U. A. M.

Posner, G. J.; Strike, K. A.; Hewson, P. W., y Gertzog, W. A. (1982). Acommodation of a scientific conception: toward a theory of conceptual change. Science Education, 66 (2), 211-227.

Pozo, J. I. (1989). Teorías cognitivas del aprendizaje. Madrid: Morata.

Pozo, J. I., y Carretero, M. (1987). Del pensamiento formal a las concepciones espontáneas. ¿Qué cambia en la enseñanza de la ciencia? Infancia y Aprendizaje, 38, 35.52.

Pozo, J. I., y Carretero, M. (1992). Causal theories and reasoning strategies by experts and novices in Mechanics. En A. Demetriou, M. Shayer y A. Efklides (Eds.), Neopiagetian theories of cognitive development: implications and applications. Londres: Routledge and Kegan Paul.

Pozo, J. I.; Gomez Crespo, M. A.; Limon, M., y Sanz, A. (1991a). Procesos cognitivos en la comprensión de la ciencia: ideas de los alumnos sobre la química. Madrid: Servicio de Publicaciones del M. E. C.

Pozo, J. I.; Sanz, A.; Gomez Crespo, M. A., y Limón, M. (1991b). Las ideas de los alumnos sobre la ciencia: una interpretación desde la psicología cognitiva. Enseñanza de las Ciencias, 9 (1), 83-94.

Pozo, J. I.; Pérez Echeverría, M. P.; Sanz, A., y Limon, M. (1992). Las ideas de los alumnos sobre la ciencia como teorías implícitas. Infancia y Aprendizaje, 57, 3-22.

ResNICK, L. (1989). Introduction. En L. Resnick (Ed.), Knowing learning and instruction. Hillsdale, N. J.: LEA.

Rivière, A. (1987). El sujeto de la psicología cognitiva. Madrid: Alianza.

Rodrigo, M. J. (1985). Las teorías implícitas en el conocimiento social. Infancia y Aprendizaje, $31.32,145-156$.

Rosch, E. (1978). Principles of categorization. En E. Rosch y B. Lloyd (Eds:), Cognition and categorization. Hillsdale, N. Y.: Erlbaum.

Rosch, E. (1983). Prototype and logical classification: the two systems. En E. K. Scholnick (Ed.), New trend in conceptual representation: challenges to Piaget's theory? Hillsdale, N. J.: L.E.A.

Sternberg, R. J., y Frensch, P. A. (eds.) (1991). Complex Problem Solving. Hillsdale, N. J.: L.E.A.

Tweney, R. D.; Doherty, M. E., y Mynatt, C. R. (eds.) (1981). On scientific thinking. Nueva York: Columbia University Press.

Vygotsky, L. S. (1934). Mysblenie i rech. Trad. cast de M. M. Rotger. Pensamiento y Lenguaje. Buenos Aires: La Pléyade, 1977. 
WELLINGTON, J. (Ed.). (1989). Skills and processes in science education. Londres: Routledge. Wertheimer, M. (1945). Productive thinking. Chicago: Chicago University Press.

WEst, L. H. T., y PINES, A. L. (Eds.) (1985). Cognitive structure and conceptual change. Orlando: Academic Press.

Yates, J.; Bessman, M.; Dunne, M.; Jertson, D.; Sly, K., y Wendelboe, B. (1988). Are conceptions of motion based on a naive theory or on prototypes? Cognition, 29, 251-275.

\section{Extended Summary}

In the last two decades there has been a striking growth of research on Science learning and teaching processes. Although the psychological processes involved in scientific thought have been a continuous area of interest for psychologists (e.g., Vygotsky, 1934; Wertheimer, 1945; and above all Piaget's impressive work on Genetic Epistemology), it is only in recent years that there has been a similar concern among science teachers. Through the constructivist viewpoint, teachers and researchers in general concerned with science education discovered the relevance of pupils' ideas and skills to the science curriculum. Recent research guided by didactic concerns has mostly centred on the study of students' 'alternative frameworks' on the main concepts of the science curriculum (e.g., Driver, Guesne, and Tiberghien, 1985; Lucas and Black, 1991). This research has focussed on specific areas of the science curriculum and has been essentially descriptive in nature, providing us with detailed knowledge of students' ideas versus those defended by modern scientists.

Recent research work from a psychological perspective has been mostly based on the concepts and concerns developed by Cognitive Psychology. Psychological studies adopting an instructional approach (e.g., Lesgold \& Glaser, 1989; Resnick, 1989) have been comparatively more interested in expert performance during science problem solving than in novices or students' ideas about science.

Therefore it is neccesary to outline the main differences between psychology oriented and teaching oriented research. Psychological studies are concerned with the cognitive processes involved in science problem solving, whilst didactic research has focussed on students' specific ideas (or 'alternative frameworks' or 'misconceptions'). Apart from its more general process orientation, psychological research is more centred in expert or scientist's performance than in analysing children and adolescents' science learning difficulties. Finally, both approaches differ in their methodologies and data analysis criteria.

Inspite of these outstanding differences both approaches to science learning and teaching processes share a constructivist conception of learning. This implies a shared concern for the cognitive processes involved in students' science learning and understanding. Thus, different perspectives have not prevented Psychology and Didactics to obtain similar research results and to share common concerns. Psychological research together with work on students' 'alternative framework" have obtained similar results on the cognitive -or what Bachelard calls 'Epistemological' - obstacles which people come across when they try to understand and think about scientific content. These cognitive constraints give rise to students' 'intuitive science' or 'alternative frameworks'. They have been identified, not only in students or in novice subjects, but in some cases also in expert scientific thought (e.g., Tweney, Doherty, and Mynatt, 1981; Kahneman, Tversky, and Slovic, 1984).

For this reason, it is necessary to apply recent cognitive-instructional re- 


\section{4}

search results to analysing educational research on students' ideas. Beyond constructivism, both approaches are not only different but in many ways also complementary. The theoretical and methodological weaknesses of studies on students' 'alternative framework' can be partly overlooked through the achievements of cognitive psychologists (e.g., the concept of 'mental model' or 'implicit theory" used to analyse students' ideas about science). Psychological research, however, should take into account the educational relevance and meaning of 'alternative framework' studies (e.g., task content and setting should be determined by curricular concerns and not just by theoretical criteria).

Beyond this reciprocal support, both approaches must cooperate if they are to answer two basic questions which both psychologists and science teachers are asking themselves. The first question relates to the processes involved in conceptual change (e.g., Carey, 1985; Gil and Carrascosa, 1985; Hashweh, 1986; Hewson and Hewson, 1987; Posner et al., 1982; Pozo, 1989; West and Pines, 1985). Secondly, more research is necessary to uncover how students change their conceptions or how novices become experts. Finally, both approaches are also increasingly interested in contextual variables that affect the activation of science knowledge in both academic and everyday settings (Claxton, 1991; Pozo et al., 1992). There is a common trend towards 'situated learning' (e.g., Lave and Webner, 1981) in the study of science learning and teaching. This common trend perhaps indicates where the immediate future of research in this area lies. 